\title{
Moderne Endoskope eröffnen neue Perspektiven
}

\author{
Die Endoskopie hat sich in den letzten Jahrzehnten von einem rein diagnostischen \\ Verfahren zur Detektion von Schleimhautveränderungen zu einem therapeutischen \\ Instrument weiterentwickelt, das sogar operative Eingriffe ersetzen kann.
}

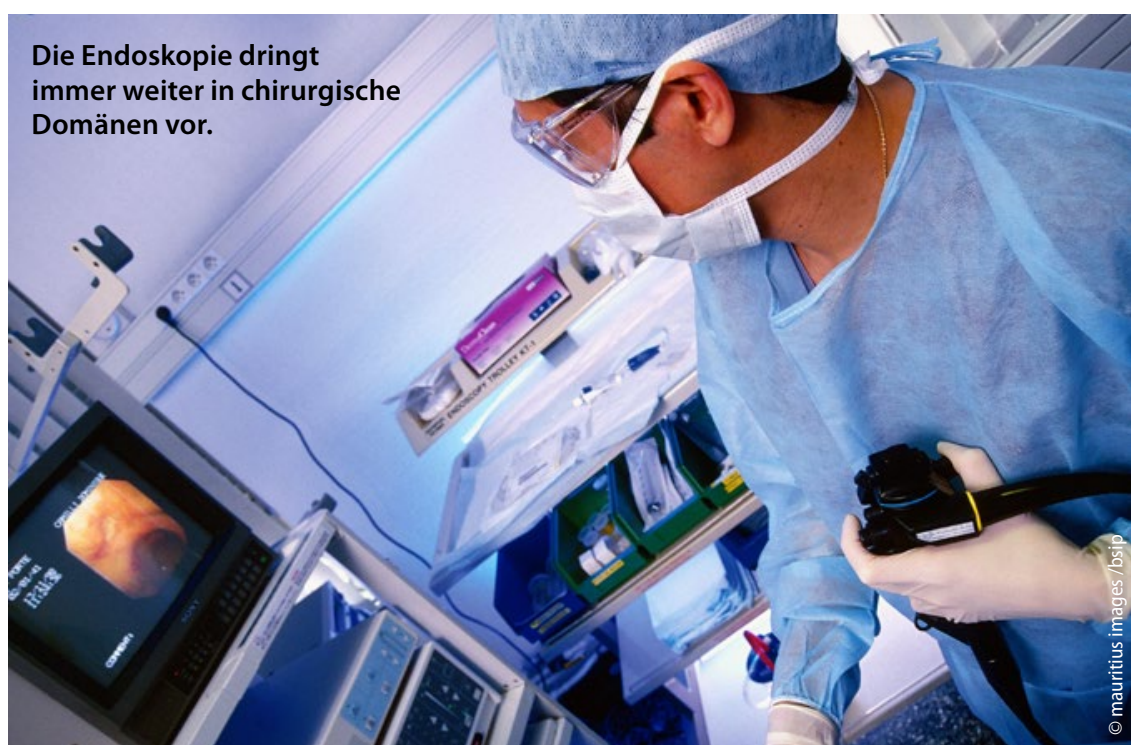

„Längst ist die diagnostische Endoskopie in den Kosmos der Endomikroskopie vorgedrungen“, sagte Dr. Torsten Beyna, Leiter der Endoskopie am Evangelischen Krankenhaus in Düsseldorf. Auch habe die interventionelle Endoskopie die natürliche Barriere der Wand des Magen-Darm-Traktes hinter sich gelassen und damit ihre Indikationen auf Gebiete ausgedehnt, die früher traditionell der Chirurgie vorbehalten waren. „Die Endoskopie ermöglicht eine patientenorientierte individualisierte Therapie nach dem Prinzip: So radikal wie nötig, so schonend wie möglich“, so Beyna.

\section{Höhere Ausbeute durch moderne Technologie}

Voraussetzung für eine noch effektivere Vorsorge im Rahmen der Früherkennung von Krebserkrankungen und deren Vorstufen sind innovative Endoskopiesysteme mit Weitwinkeloptik und virtuellen Filtersystemen. Mit Hilfe modernster hochauflösender Magnifika- tionsendoskope (Zoom-Endoskopie) mit computergestützten optischen Färbeverfahren (virtuelle Chromoendoskopie) evtl. ergänzt durch die hochauflösende endoskopische Ultraschalluntersuchung gelingt heute in den meisten Fällen eine zuverlässige Charakterisierung der Läsion bzgl. Dignität - unabdingbare Voraussetzung für die Wahl des richtigen therapeutischen Verfahrens. Dazu gehören die endoskopische Mukosaresektion, die endoskopische Submukosadissektion und neuerdings die endoskopische Vollwandresektion, bei der vollständige Teile der Wand des Magen-Darm-Traktes entfernt werden.

\section{Endoskopische Therapie der Refluxkrankheit}

Unbestritten sind Protonenpumpeninhibitoren (PPI) Standard bei der Therapie der Refluxkrankheit. Wenn aber diese Therapie nicht ausreicht oder nicht vertragen wird und der Patient sich keinem chirurgischen Eingriff unterziehen möchte, sollte eine endoskopische Therapie diskutiert werden. „Mit dem sog. MUSE-Verfahren, einem endoskopischen ultraschallkontrollierten Klammernahtgerät, steht erstmals ein modernes rein endoskopisches Verfahren zur Verfügung, das ein der operativen Fundoplicatio gleichwertiges Langzeitergebnis verspricht", so Beyna.

Auch Patienten mit einer schweren Achalasie, bei denen die Ballondilatation kein zufriedenstellendes Ergebnis zeigt, kann heute auf endoskopischen Weg geholfen werden, und zwar mit der peroralen endoskopischen Myotomie (POEM). Dabei wird endoskopisch eine kleine Öffnung an der Schleimhaut des Ösophagus angelegt und dann endoskopisch unter der Schleimhaut ein Tunnel bis zum Mageneingang geschaffen. Von diesem Tunnel aus wird dann der Muskel im Bereich des untern Ösophagussphinkters schrittweise gespalten.

\section{Bahnbrechende Neuerfindung: Spiralenteroskopie}

„Eine bahnbrechende Neuentwicklung ist die jetzt erstmals am Menschen eingesetzte motorisierte Spiralenteroskopie“, so Prof. Horst Neuhaus, Chefarzt der gastroenterologischen Abteilung am Evangelischen Krankenhaus in Düsseldorf. Bei diesem Verfahren wird eine sich drehende wechselbare Spirale auf ein wiederverwendbares flexibles Endoskop montiert, womit der Dünndarm quasi aufgerollt wird. Nach ersten Erfahrungen kann damit innerhalb kürzester Zeit ein Großteil des Dünndarms eingesehen und für therapeutische Zwecke erreicht werden.

\section{Dr. Peter Stiefelhagen}

- Pressekonferenz im Rahmen des 18. Internationalen Endoskopie Symposiums, 11.2.2016 in Düsseldorf 